\title{
VISUAL-SIMULATION-BASED PERSONALIZED GARMENT BLOCK DESIGN METHOD FOR PHYSICALLY DISABLED PEOPLE WITH SCOLIOSIS (PDPS)
}

\author{
Yan Hong ${ }^{1,2,3}$, Pascal Bruniaux ${ }^{2}$, Xianyi Zeng ${ }^{2}$, Kaixuan Liu ${ }^{2}$, Antonela Curteza ${ }^{3}$, Yan Chen ${ }^{1}$ \\ ${ }^{1}$ College of Textile and Clothing Engineering, Soochow University, Suzhou 215021, China \\ ${ }^{2}$ GEMTEX, ENSAIT, 2 allée Louise et Victor Champier, 59056 Roubaix Cedex 1, France \\ ${ }^{3}$ Technical University of lasi, Dimitrie Mangeron Bd., 53, lasi -700050, Romania
}

\begin{abstract}
:
This research presented a novel method using 3D simulation methods to design customized garments for physically disabled people with scoliosis (PDPS). The proposed method is based on the virtual human model created from $3 D$ scanning, permitting to simulate the consumer's morphological shape with atypical physical deformations. Next, customized $2 D$ and $3 D$ virtual garment prototyping tools will be used to create products through interactions. The proposed 3D garment design method is based on the concept of knowledge-based design, using the design knowledge and process already applied to normal body shapes successfully. The characters of the PDPS and the relationship between human body and garment are considered in the prototyping process. As a visualized collaborative design process, the communication between designer and consumer is ensured, permitting to adapt the finished product to disabled people afflicted with severe scoliosis.
\end{abstract}

\section{Keywords:}

Virtual simulation, disabled people, atypical morphotype, knowledge engineering, 3D design, ergonomics

\section{Introduction}

Scoliosis is a lateral curvature usually associated with axial rotation of the spine [1]. Compared with compared with that of normal body shape, the characters of physically disabled people with scoliosis (PDPS) are as following: (1) his/her bent spine is a curve shape instead of a straight line; (2) The shape of the upper body of a disabled person is extremely asymmetric, while the lower body, including the hip and legs, is similar to the normal symmetric posture; (3) Many positions on the body surface usually used to garment design, such as the points of the left shoulder, left chest and waist of back, are hidden inside the body surface. In this condition, the figures of the disabled people, especially the upper body, don't fit into the standard sizes, used for garment design and production available in the stores, which leads the disabled people PDPS into the consumer market with special needs in terms of personalized products [2].

Usually, adapting ready-to-wear (RTW) garments is widely applied solution for satisfying the personalization requirement. However, the adaption not only takes time for production but also cannot ensure a good comfort and fit [3]. The deformation of the body shape represents a complex geometry, which cannot apply the traditional 2D garment design process and related design knowledge, as the foundation of the $2 \mathrm{D}$ garment design process is based on the agreement of the spin is straight. There is no efficient design solution for PDPS that is available in industry. The personalization for PDPS requires more requirements of the technical part in the design process, which is even far more complex than other disabilities [4].

There are two garment design methods, using in fashion industry: 2D-to-3D and 3D-to-2D approach [5]. 2D-to-3D approach is based on the several sizing rules, using the measurements of the standard body, which can be recognized as a conventional garment design method. In the 2D-to-3D approach, 2D patterns are sketched manually with construction lines and darts, and later assembled through a sewing procedure to produce realistic draping simulation [6]. However, as explained before, 2D-to-3D approach is based on the agreement that the spin of the customer is straight, it cannot produce precisely fit garment for personalization [7]. In 3D-to-2D approach, the pattern pieces are generated from a $3 \mathrm{D}$ physical mannequin using fabric-draping process [8]. The draping process is frequently used by designers for quickly generating the form of a garment by mouldings, cutting and pinning fabric to a mannequin or individual [9]. Style lines and construction details of the drape are carefully marked and removed step by step. Fabric pieces with the construction and style details are generated. Darts will be generated in the same time in the previous steps. The fabric pieces are then laid to be flat and traced over a pattern paper. The pattern is finalized by adding directional marks such as grain lines, notches, buttonholes, correct seam and hem allowances and facings [10]. Compare with 2D-to-3D approach, 3D-to-2D approach seems to be a better solution for designing personalized garment for physically disabled people with scoliosis, however, the 3D-to-2D approach is based on a physical mannequin, which is impossible to be obtained for physically disabled people with scoliosis.

Computer aided design technology (CAD) is blooming rapidly in fashion industry, especially in personalized garment design area. Computer aided design technology (CAD), as a visualized design technology, ensures a communication platform between designers and consumers, which is frequently required by the personalized product design [11]. There are several 
new Computer aided design technologies (CAD) related to personalized product design. First, 3D scanning technology permits a digitalized human model, which can serve as a visual mannequin [12]. Second, virtual simulation technology provides the technical solution to modeling the complex geometry [13]. Third, the visual try-on technology permits fast validation of design ideas and principles [14]. These technologies seem to be efficient solution to design for PDPS [15].

Actually there are some research results using the previous Computer aided design technology (CAD) to create garment products directly [2]. Using 3D scanning, a digitalized human body model can be obtained, based on which the visual prototyping can be realized. The wearing ease and garment style can be controlled in the design process by adjusting the distance between the garment surface and the corresponding area of the human body. It provides technical solution for designing customized garment for physically disabled people with scoliosis. However, the processing procedure of this method is rather time consuming. The precision of the design effect depends on the quantity of the wearing ease control points, which contains intensive manual work. Also, only garment with simple style can be realized using this method. The design cases are rather independent, which cannot meet the requirement of mass production. Garment block is the primary design of a garment, which can be further extended to various styles with different design elements [16]. The block plays an important role in 2D-to-3D design process [17]. In practice, most of the ready-to-wear garments are developed from the garment block [18].

In this context, a personalized garment block design method is proposed in this paper, using the virtual simulation technology, based on the digitalized human body model created from 3D scanning. Relationship between garment block and human body is investigated to provide reference in the design process. Visual try-on technology will be applied in the evaluation design effect to ensure the desired design ideas. The proposed garment block can be further applied to mass production. Comfort and fit can be fully considered in the design process, which ensures the personalized design for physically disabled people with scoliosis.

The rest of the study is organized as follows. The proposed design method is introduced in the second part. An experiment investigating the relation between the garment block and human body is presented in the third part. A case study is discussed in the following part. Then, a discussion section is proposed to compare the proposed design method with current design method. The final section concludes the paper.

\section{Experiment}

\subsection{General Scheme}

In this study, a visual simulation based design method is proposed to develop a customized human centered 3D flattenable garment block with controlled ease values, for disabled people with scoliosis, meeting the requirements of disfigurement and comfort. Figure 1 depicts the methodology framework, which includes 5 phases in the process. In the first phase, a digitalized human body model is triangulated by means of a number of anatomical references. In the second phase, the feature points of the body surface, including the anatomical landmarks and the body features, are extracted, referring to that of conventional 3D-to-2D design method. As discussed in our previous study, ease values are volumes of the gap between the feature points for garment design on the human body and the corresponding feature points on the garment surface. Previous studies have been done to investigate the volumes of the gap [11]. In this research, wearing ease values at different positions are then accurately determined based on our previous research result and carefully distributed into feature lines of the garment to be designed, in order to establish a 3D wireframe. In the third phase, the 3D wireframe is established from the previous phase. In the fourth phase, the 2D block patterns are finally flattened and adjusted from the 3D garment blocks. The evaluation and adjustment based on $3 D$ virtual try-on is performed repeatedly to obtain a satisfying final result.

The proposed design method has the following contributions related to the current garment design situation.

1) The proposed design method provides technical solution to personalized garment design for physically disabled people with scoliosis. The method design from personalized garment block, which can be further applied to design all kinds of garment products. Personalized mass production can be realized using the proposed method.

2) Designing from garment block can significantly reduce the time used in the design process in the long term, compared to modeling the personalized garment product directly.

3) Using 3D simulation and virtual try-on, a collaborative design platform can be realized. It is possible to set up a quick and easy interaction between the $2 \mathrm{D}$ technical space of the garment (flat patterns and other technical parameters such as colour and texture) and the 3D perceptual space of the finished product. Also, the communication between designers and customers can be realized.

\subsection{Method}

In this section, an experiment was carried out to investigate the relationship between the garment block and human body, in order to provide the design reference for the design process for designing for disabled people. Wearing ease and the contact points between the garment block surface and human body surface will be studied in the experiment.

A mannequin (type of Missy 10 from Alvaform Company) was prepared. First, a measurement process about the chest circumference, waist circumference and back length, of the Missy 10 mannequin was performed meeting the requirements of a garment block design. Then, a set of garment block patter using BUNKA style for the Missy 10 mannequin was designed [19]. For clearly observation, different from the conventional 
garment block, the wearing eases of the chest and waist were given 0 . After that, the modified garment block was copied to a transparent paper for the try-on. Figure 2 presents the try-on result.

It can be concluded: (1) Neck circumference and waist circumference are completely fit to that on the mannequin. (2) Bust circumference is completely fit to the body except for the breast points, especially the armpit circumference and under chest circumference. (3) The surface of a garment block without wearing ease in chest and waist can be regarded as a 3D shape, developing from the shoulder line, side body line, waist curve and neck curve, which are contact directly onto the human body surface. The unfit here is because the breast is convex area [20]. To get the pattern of a convex area, the geometric principle of "Angular Defect" from Geometry can be applied [21]. For a 3D shape created with a vertex and several corresponding facets, a plate plane with a defected angular,

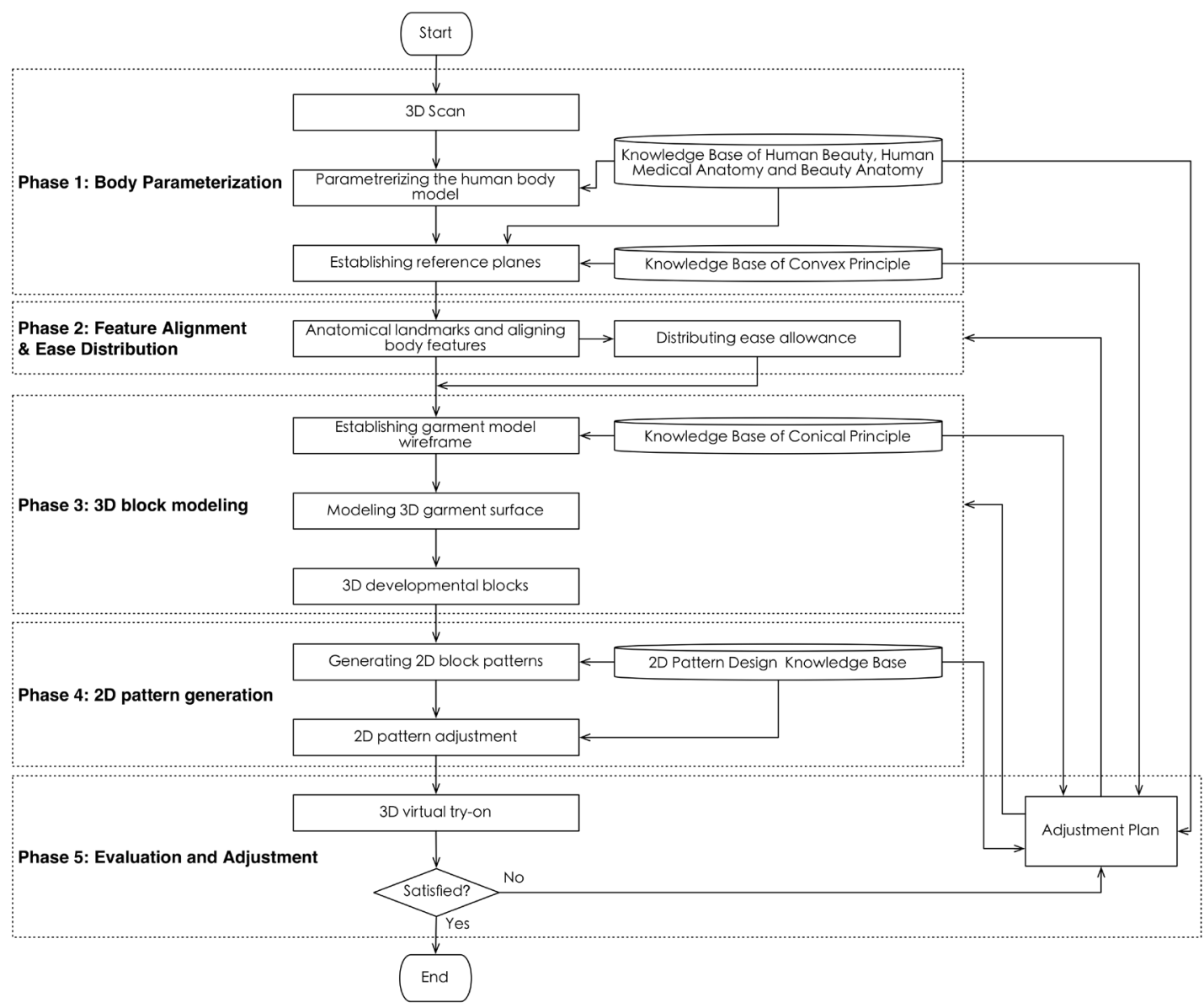

Figure 1. General scheme for design of a customized human centred 3D flattenable garment block

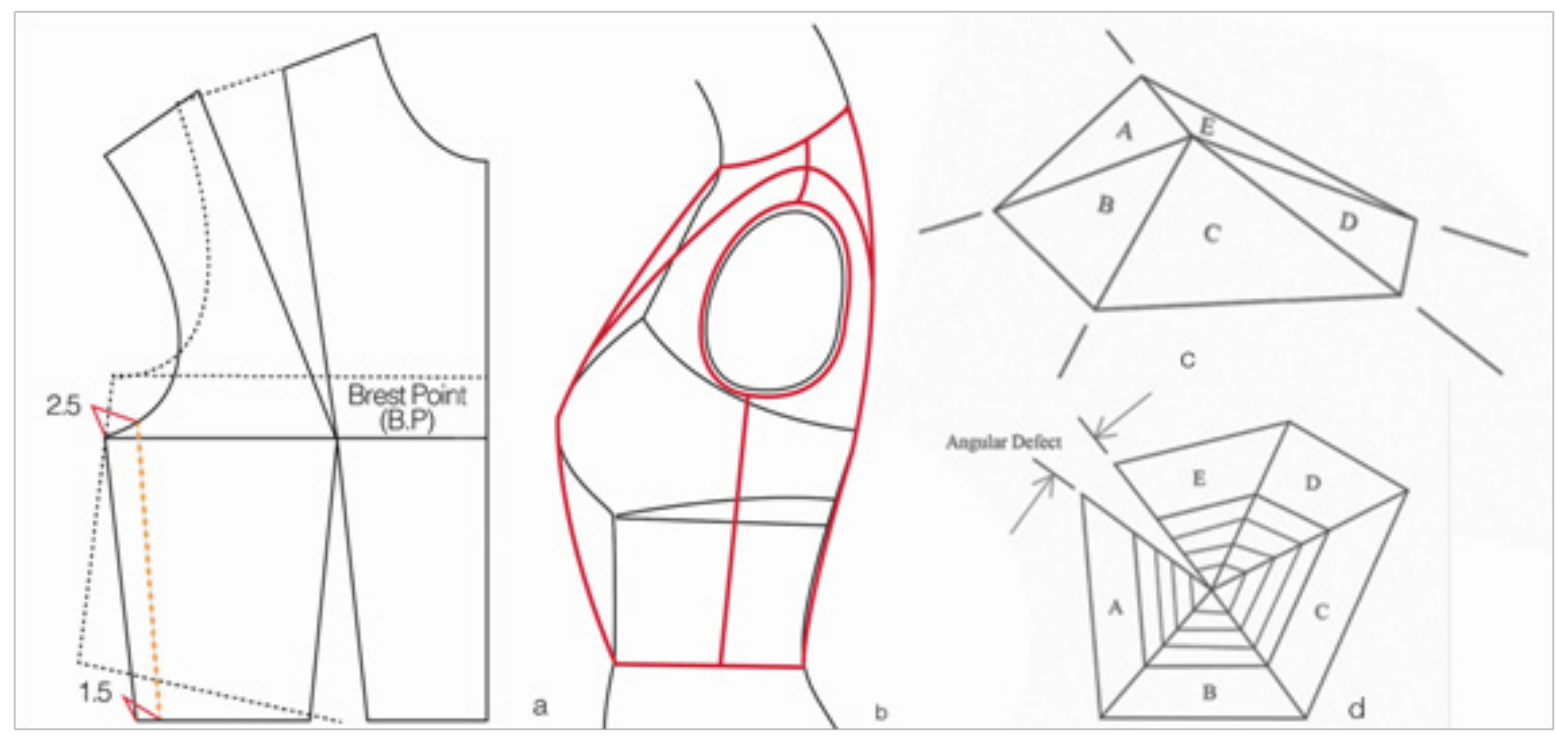

Figure 2. (a) Garment block pattern without wearing ease in chest and waist, (b) wearing effect of the modified garment block, (c) vertex on 3D surface, (d) vertex flattened to give angular defect 
which contains several flattened shapers exactly the same with these facets, will generally exist in order to perfectly cover the surface of the shape, shown in Figure 2-c, d [21].

Using the conclusion of the experiment, the visual simulation based design method for PDPS can be realized. First, the feature points of the human body surface used for further prototyping should be detected. Then following the general from of a garment, the contact construction lines and cures, such as neck curves, shoulder lines, side lines, armhole curves, waist curves can be draw on the surface of a mannequin. After that, an accelerated surface can be applied to modeling this surface, developing from the lines and curves mentioned before, which has a tangent point via the breast point [18]. The general idea of the simulation process is shown in Figure 3.

\subsection{Case study}

A case study using the proposed design method to design a personalized garment block for the PDPS is presented in this section. A case of application using the proposed garment block is also introduced.

\subsubsection{Body parameterization}

In this study, a digitalized human body was prepared to serve as a personalized visual mannequin, with which the design and construction of the personalized garment can be realized [22].

First, a 3D scanning operation was performed to get the raw data, which is a set of points forms the shape of the body surface of the scanned human body. The scanning operation was performed using 3D scanning machine and corresponding software from Human Solution company. Next, scanned data from 3D scanning, which consists of a three-dimensional point cloud of the object surface was then meshed into small facets, using the meshing tool of the Rapidform software. The meshed human body model was then exported into Lectra Design Concept software, which is able to edit 3D objects. The software chosen here are of commercial type, which are wildly used in fashion industry. These facets can be regarded as the sub surface of the body, which can be modified. The number of the facets will determine the quality of the virtual body surface. The identification of body features can be conducted with the digitalized human body model [23].

Then several reference planes were established to better observing the body shape. The asymmetric deformation makes it impossible to establish standard Transverse Plane, Sagittal Plane and the Coronal Plane that used for people with normal body shape [24]. However, as the shape of the lower body, including the hip and legs, is symmetric, it provides a possibility of establishing reference planes, similar to those of people with standard body shape. For this purpose, we establish the same Transverse Plane, Sagittal Plane and the Coronal Plane as people with standard body shape (see as the yellow lines in Figure 4), which makes it of great significance to provide reference comparing the convex points and areas [25]. Using the reference planes for the lower body, designers can observe the human body in different views in order to identify the feature points of the human body used for virtual prototyping.

\subsubsection{Feature alignment and ease distribution}

The feature points of a garment can be developed from the feature points of the human body. Following that of people with normal body shape, feature points of the human body used for prototyping are detected, using the experience of the designer. These feature points are breast points, shoulder points, scapular points, neck points, cross points of waistline and sideline, as presented in Figure 3.

The wearing ease should be discussed first for acquiring the feature points of the garment. In the design process, the wearing ease is generally determined by the relationship between the garment and the human body. If the design is aimed at showing more beauty of the human body, the value of the wearing ease can be smaller. If the design is aimed at showing more beauty of the garment, especially its draping effects, the value of the wearing ease can be bigger. For the garment position close to the body deformation, the value of wearing ease can be bigger,

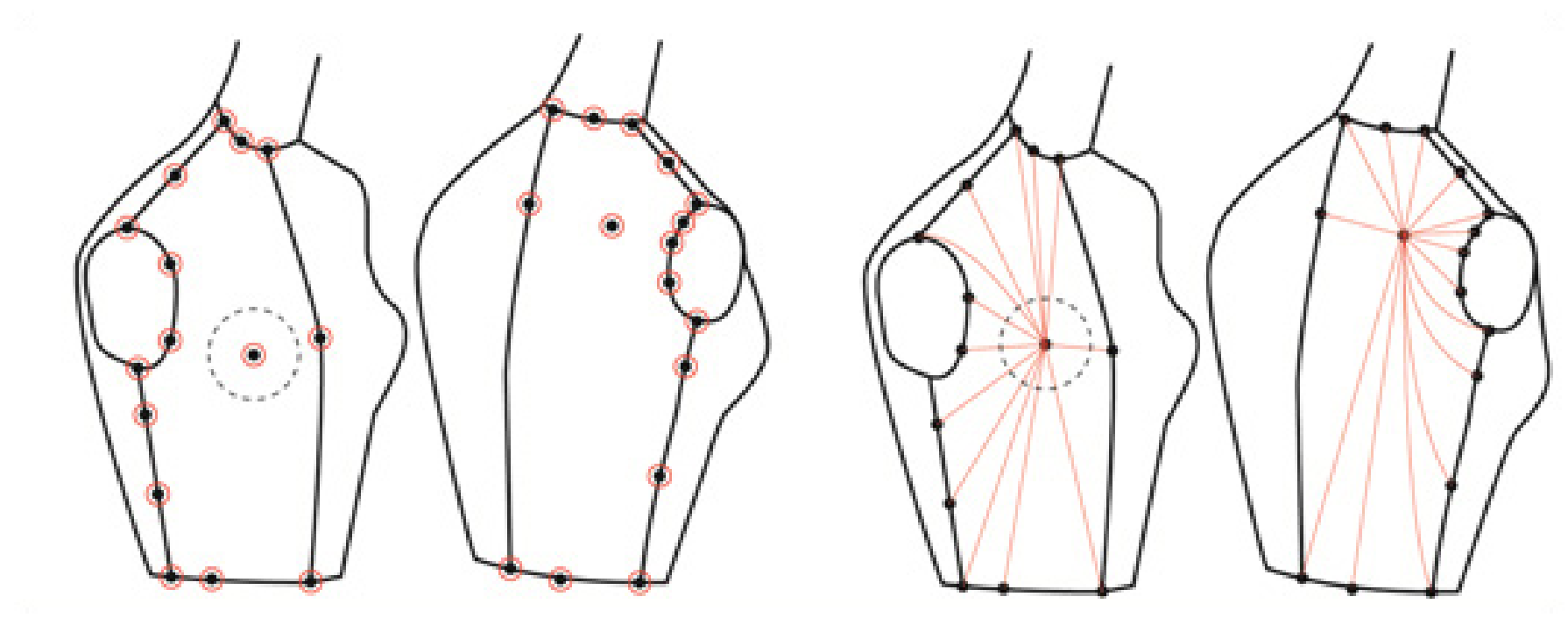

Figure 3: Design related feature points on the human body surface 


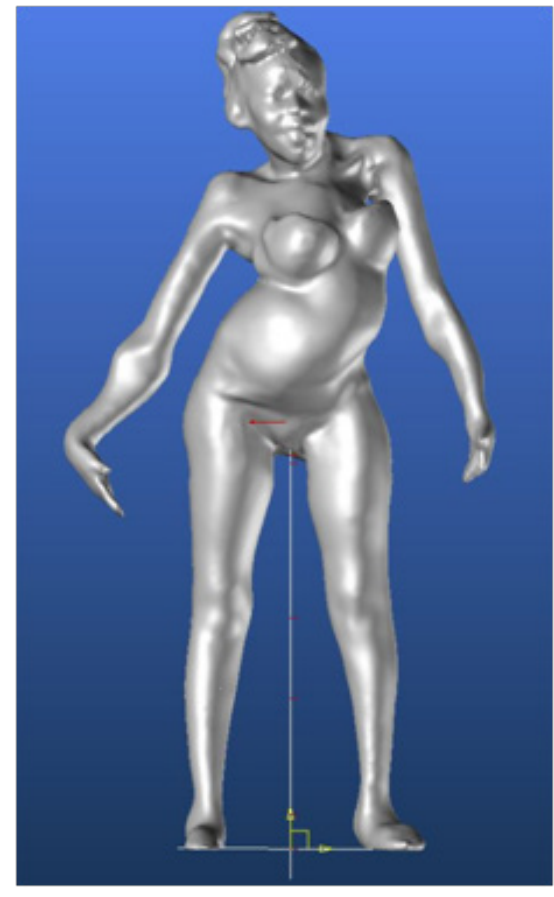

Figure 4. Reference axis for the people with scoliosis

while it can be smaller if we wish to show the curve of a specific body part.

In this context, the entire feature points of the human body are classified depending on the shape of the area it exists compared with that of normal body shape. If the feature point is more convex than that of normal body shape, such as the scapular points, it will be processed using the same method when designing for the breast points of people with normal body shape. If the feature point is more sunken, more ease allowance will be given than that used to design for normal body shape, such as the cross points of waistline and sideline, using the professional knowledge of designer. After running the procedure of Design - Display - Evaluation - Adjustment for several times, a suitable wearing ease can be determined for a desired fit effect [26].

\subsubsection{D block modeling}

After the previous step, all the feature points of the desired garment block, which permit to determine the structure outline of the virtual garment block, can be obtained. Then, the structure outline is drawn using fashion design knowledge. First, one line is drawn between the two breast points to probe the middle point. This point will be then linked with the front neck point and the central point of the waistline to determine the front central line. For the back central line, the same operation will be carried out. Because of the deformation of the left waist and the left shoulder, one line is drawn between the left armhole point and the left waist point; another line is drawn between the left shoulder point and the left neck point.

The feature points of the human body that contact to that of the garment surface will be linked as line or curves following the framework used for normal body shape. These curves and lines are neck curves, shoulder lines, sidelines, front and back lines. Several controlling points will then be manually positioned on the lines defined previously in order to mess the wireframe of the garment surface. Classical 2D pattern design knowledge will be applied in this process. The number and positions of the controlling points are determined according to the reference of people with standard body shape related to Figure 3 . Then a set of curves will be drawn between the convex points (breast points and scapular points) and the controlling points to form the wireframe of the garment surface. After that, following the process in Figure 3, the garment block surface can be created. Figure 5 presents some details in designing the garment block framework.

The wireframe of the garment block is then modelled by triangulating and assembling different parts bounded by the deformed wireframe. The technical method follows the same principle of digitalized human body modelling using the RapidForm software. Figure 6-a shows the meshed 3D garment block surface.

\subsubsection{D pattern generation}

The meshed garment block surface can then be applied to generate the corresponding $2 \mathrm{D}$ patterns using the flattening operation. The generation of flattened $2 \mathrm{D}$ patterns also strictly follows the principle classical 2D pattern design knowledge. Darts, folds, opening, fabric direction (Warp and Weft direction) and other important 2D pattern design elements should be fully considered. Then the meshed virtual garment surface is divided into 4 parts: right front, left front, right back and left back (Figure 6-b). 8 different surfaces are generated: 4 in the
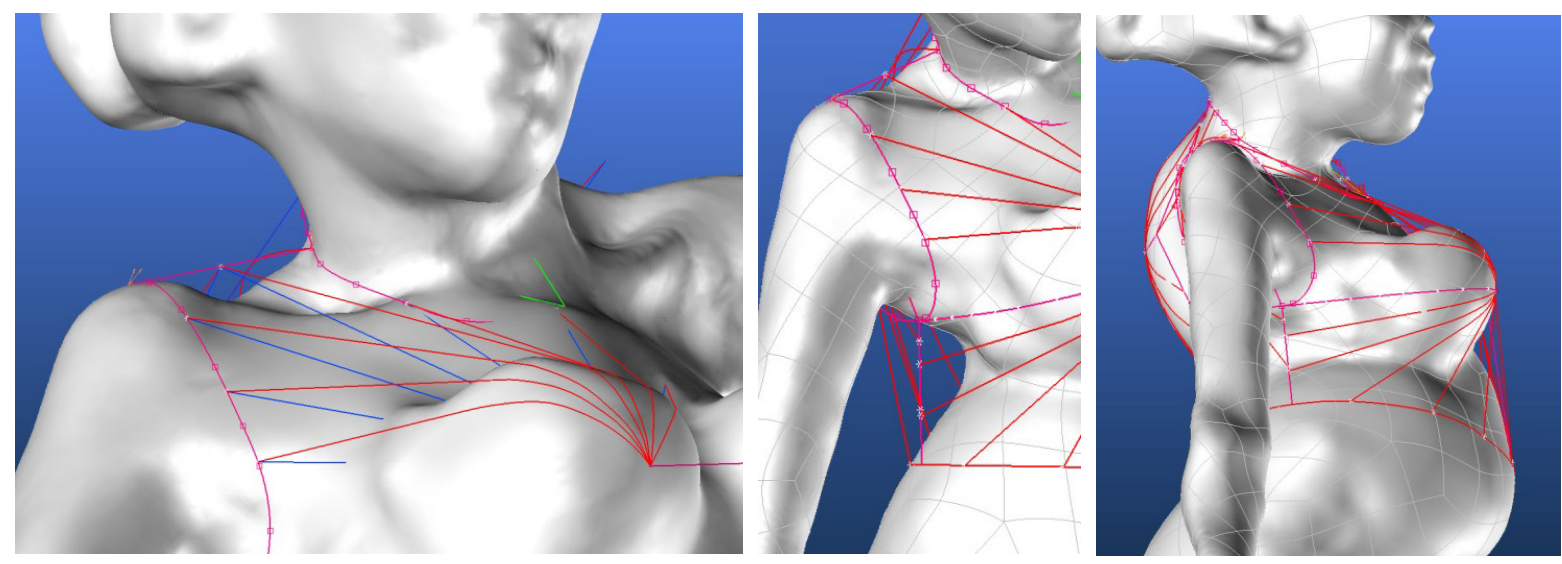

Figure 5. Details for the allowances controlling for different body areas 
front and 4 in the back. Then the 2D patterns can be flattened automatically and easily.

In Modaris software, virtual fabric can be simulated based on the parameters that of the real fabric. Using the simulated virtual fabric, a virtual try-on can be ensured to validate the design idea. There is a database of the virtual fabric, which contains common fabrics widely used in fashion industry. In this research, parameters of the selected fabric is shown in Table 1. Using these parameters, the virtual try-on with desired fabric can be realized. Figure 8 shows the flattened 2D patterns and the 3D virtual try-on in Modaris software. It should be noted that, the $2 \mathrm{D}$ patterns have already linked with the $3 \mathrm{D}$ virtual try-on. The $3 \mathrm{D}$ virtual try-on can display the modification and adjustment of the 2D patterns automatically. Table 1 presents the fabric parameters in the virtual try-on procedure.
After that, in order to make sure that the flattened 2D garment pattern can be applicable for industrial application, several modifications were performed following $2 \mathrm{D}$ pattern design knowledge. Curves of the patterns are reshaped to be smooth. Corresponding curves pairs are carefully checked to ensure the same length and in right position.

\subsubsection{Evaluation and adjustment}

The visual evaluation analysis of the proposed garment block pattern is carried out using the colorization tools of CAD software (Modaris and Lectra Design Concept) to evaluate garment pieces. Colorization can be done on several patterns of a garment selected during the activation of these tools, see Figure 8. A scale of colors is associated with each type of colorization, which can be described in Table 2.
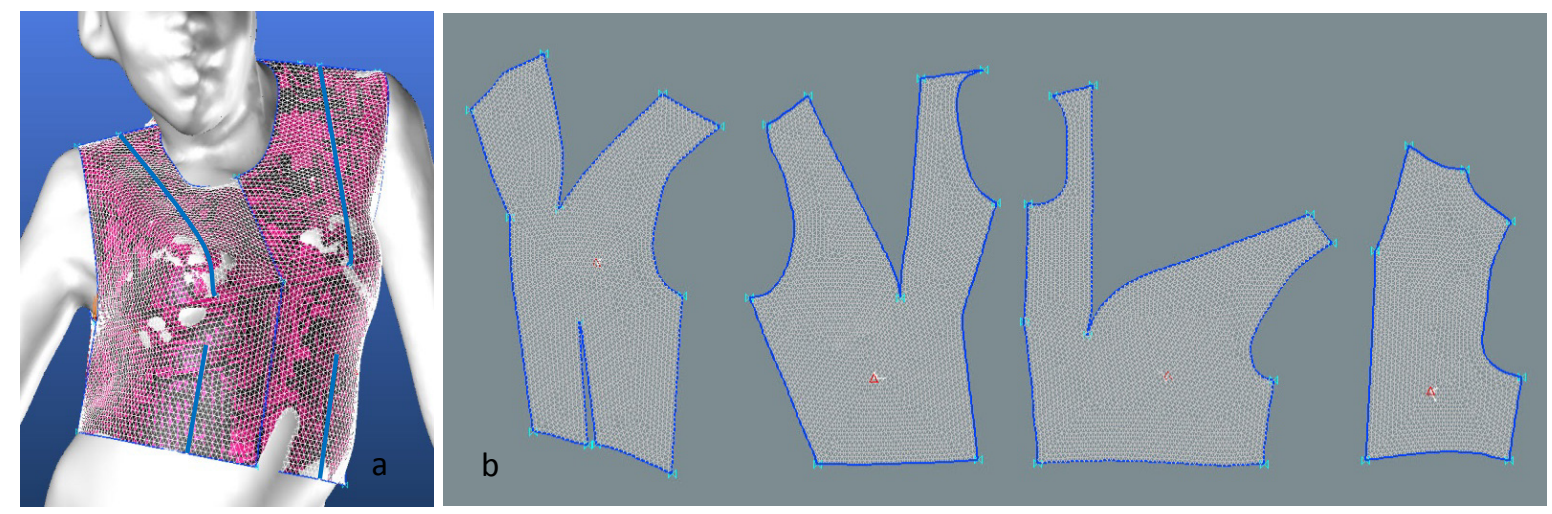

Figure 6. (a) Meshed garment block surface, (2) 2D garment patterns

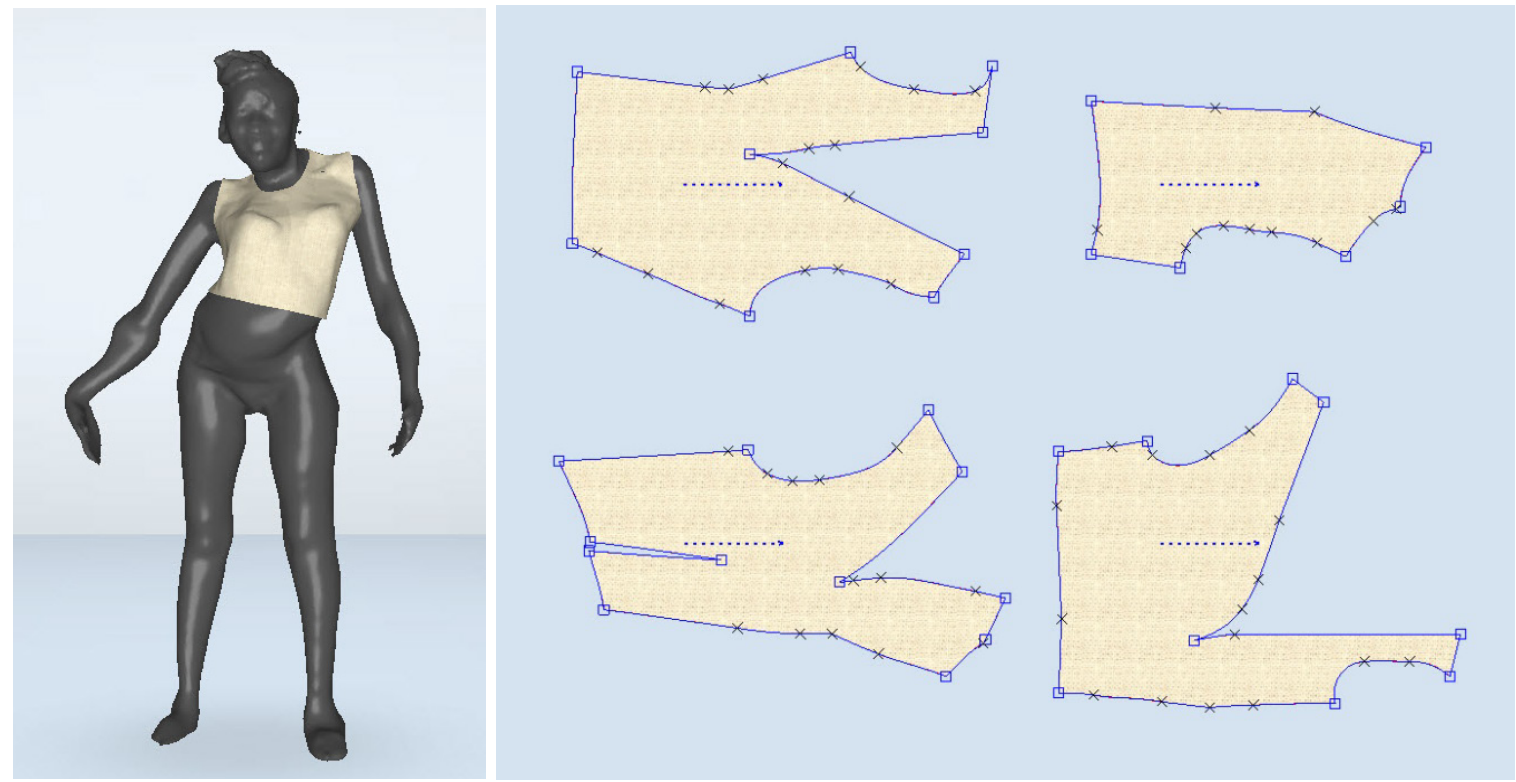

Figure 7. 3D garment block with fabric parameters and flattened 2D patterns

Table 1. Fabric parameters in the virtual try-on procedure

\begin{tabular}{|c|c|c|}
\hline Fabric parameters & Wrap & Weft \\
\hline Raw material & Polyester $60 \%$ Cotton $40 \%$ & 50 \\
\hline Yarn's fineness (tex $=\mathrm{g} / \mathrm{Km})$ & 50 & 25 \\
\hline Yarn density (yarn/cm) & 30 & \\
\hline Weave structure & Plain & \\
\hline
\end{tabular}



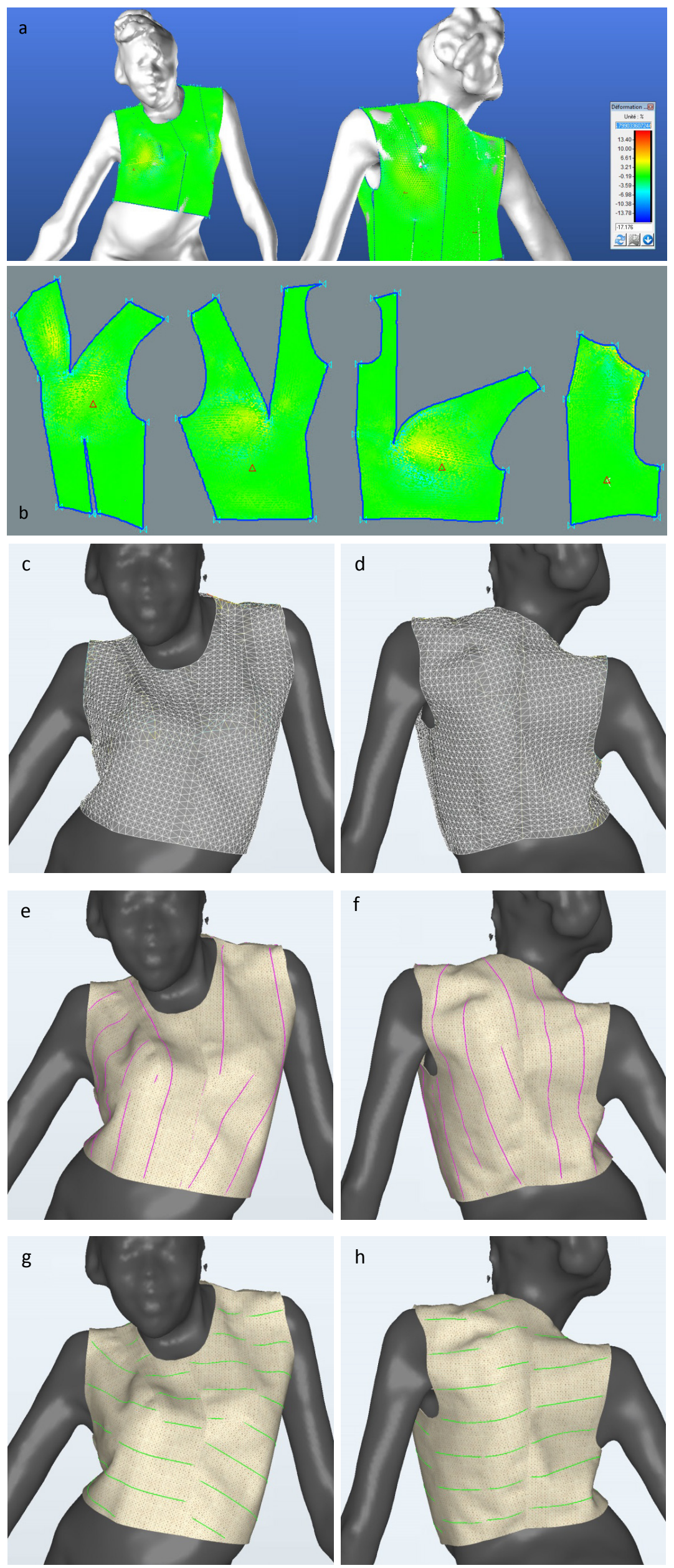

Figure 8. Pattern pieces evaluation results using the colorization tools in CAD softwares 
Table 2. The colorization tools for evaluating pattern pieces in CAD software

\begin{tabular}{|c|c|}
\hline Categories & Definition \\
\hline Ease & $\begin{array}{l}\text { Colorization of the ease on the garment permits to have an overlook of the garment ease on different } \\
\text { parts of the body. The colorization can be done on one of the garment pieces. The ease unit of measure } \\
\text { is a unit of length and corresponds to the difference between the perimeter of the mannequin and that of } \\
\text { the garment, within certain limitations. }\end{array}$ \\
\hline Mesh distortion & $\begin{array}{l}\text { This tool displays and colorizes the mesh gradually depending on its deformation. The colorization } \\
\text { occurs as a function of the deformations of sides of triangles. The deformation unit of measure is a } \\
\text { percentage. }\end{array}$ \\
\hline Warp distortion & $\begin{array}{c}\text { This tool displays the grain line deformations. The colorization occurs along the axis of the grain line of } \\
\text { the material. The deformation unit of measure is a percentage. }\end{array}$ \\
\hline Weft distortion & $\begin{array}{c}\text { This tool displays the cross line deformations. The colorization occurs along the axis of the cross line of } \\
\text { the material. The deformation unit of measure is a percentage. }\end{array}$ \\
\hline
\end{tabular}

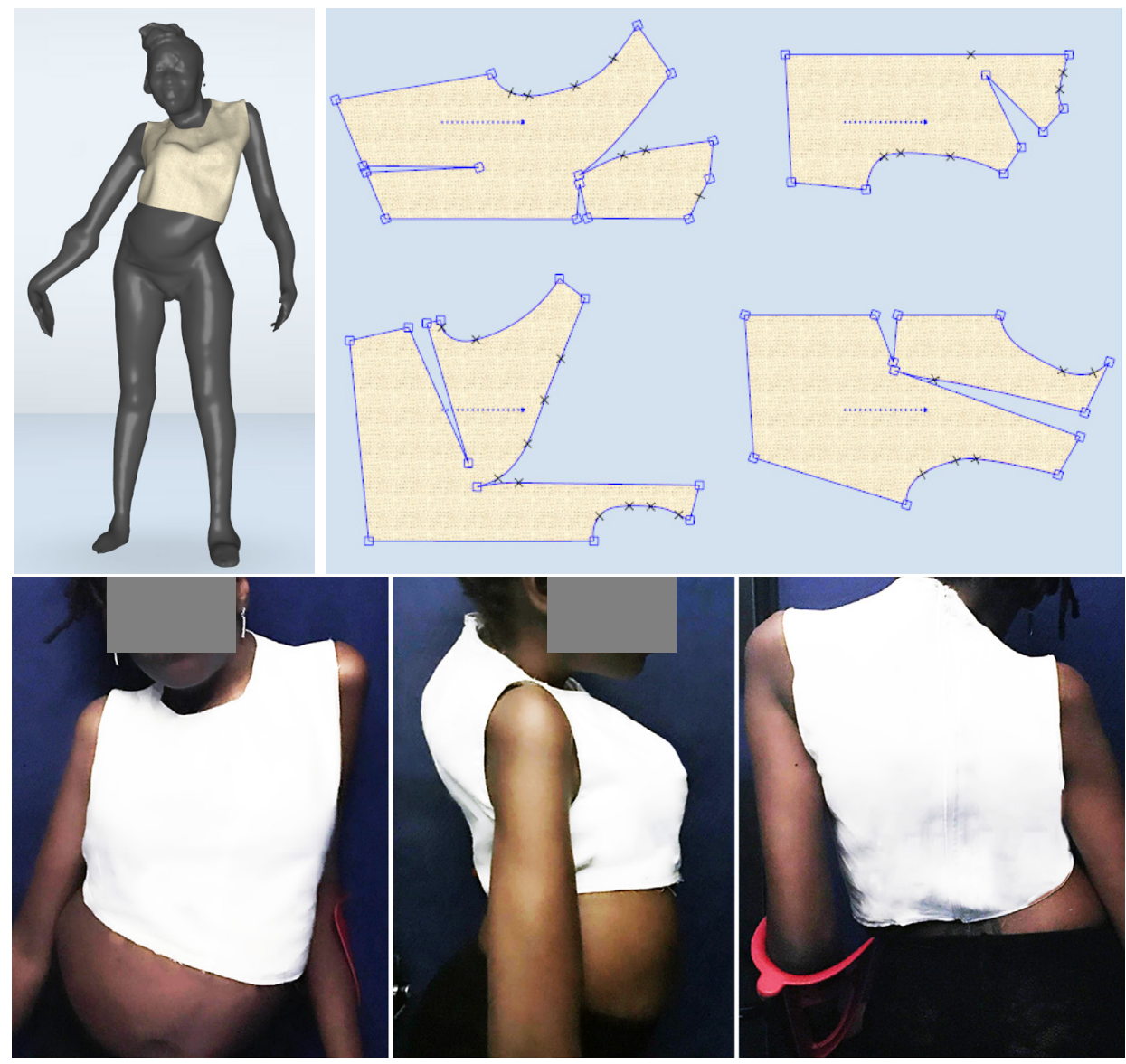

Figure 9. Final garment block (real and virtual try-on) and corresponding 2D patterns

Using ease analysis, it can be found that, the ease value near the left breast, right breast is not enough. In terms of the mesh distortion, the surface near the left scapular is not enough. From the analysis of warp distortion and weft distortion, there are some deformation around the left breast and left scapular. After the evaluation, the designers carried out some modifications, running the sequence of Display - Analysis - Adjustment based on the experience of the designers, until a satisfying design solution is obtained. These adjustments were performed near the breasts and left scapular, giving more wearing ease and adjusting the curvatures of the curves. Figure 9 shows the final block, the corresponding 3D virtual try-on and the real garment in different views.

\section{Comparison and discussion}

In order to validate the proposed design method, a set of comparison experiment was carried out. Current methods, designing for PDPSwere discussed with the proposed method.

A set of Key Performance Indicators, focusing on quality and efficiency in garment design and production process are applied to validate the different design methods (Table 3) [27].

Five experienced fashion designers and pattern makers are invited to join in the experiments to obtain a quantitative comparison between the mentioned design solutions. A set of experiments 
using three different pattern-generating methods to perform the whole stages of the garment development (from scanning to production) have been carried out in the same working environment (equipment, software), under the same working pressure. There were training sections about the two different design methods and involved equipment and software, for all the designers and patternmakers before the experiments. These experiments were carried out after the involved people were trained of the required skills (design process, equipment and software).

The experimental data of Key Performance Indicators for the two design solutions are summed up for further discussion and comparison. In the experiment, each designer will be required to perform three different methods for three times. The requirement for the design here is a garment block with the same style, construction and finishing. The average data of the six repeated experiments are performed in Table 4.

Evidently, the proposed 3D-to-2D virtual design method is more efficient than other virtual design methods for all the performance indicators.
The indicator "Total Hours Worked including overtime" shows that, compared with other design methods, the proposed method takes less time and reduces the complexity of the operation, permitting to shorten the design and production cycle. The indicators "Efficiency of Direct Operators", "Utilization of direct operators" and "Absenteeism", jointly represent the working efficiency of the design and production, point out that the proposed design method is also more efficient than other methods for designers and pattern makers. From "Number of Direct Operators", it can be easily found out that, design method in this study occupies less human resources than design methods, which is more efficient, less complex, catering to the market.

In conclusion, compared with the proposed 3D-to-2D virtual design method, the $2 \mathrm{D}$-to-3D virtual design method is relatively uncertain and complex in its design process, and the ease controlled 3D-to-2D virtual design method is a bit more complex and time consuming. What's more, the obtained design solutions from the 2D-to-3D virtual design method are less satisfying and more complex for designers. The relationship

Table 3. Different design methods and corresponding design processes

\begin{tabular}{|c|c|}
\hline Design methods & Process \\
\hline $\begin{array}{l}\text { Ease controlled } \\
\text { 3D-to-2D method }\end{array}$ & $\begin{array}{l}\text { (1) 3D scanning to modeling the human body } \\
\text { (2) Detecting the feature points on the body surface used for virtual prototyping } \\
\text { (3) Adding more ease controlling points on the body surface based on the feature points defined before } \\
\text { (4) Determining the value of wearing ease for each points defined before } \\
\begin{array}{l}\text { (5) Creating the framework of the garment surface } \\
\text { (6) } 3 D \text { to } 2 D \text { flattening }\end{array}\end{array}$ \\
\hline $\begin{array}{l}\text { Method in this } \\
\text { research }\end{array}$ & $\begin{array}{l}\text { (1) 3D scanning to modeling the human body } \\
\begin{array}{l}\text { (2) Detecting the feature points on the body surface used for virtual prototyping } \\
\text { (3) Linking part of feature points } \\
\text { (4) Creating the framework of the garment surface which has a tangent point via the convex point (B.P. } \\
\text { and scapular point) } \\
\text { (5) } 3 D \text { to } 2 D \text { flattening }\end{array}\end{array}$ \\
\hline 2D-to-3D method & $\begin{array}{c}\text { (1) Virtual try-on of different sizes of standard basic garment patterns close to customer's body size, in } \\
\text { order to find the most appropriate sizes for performing a digital simulation of the garment } \\
\text { (2) Modification of the darts using the experience of the designers based on the experience of the } \\
\text { designers repeatedly }\end{array}$ \\
\hline
\end{tabular}

Table 3. Definition of the Key Performance Indicators for garment design and production

\begin{tabular}{|c|c|}
\hline Indicators & Definition \\
\hline $\begin{array}{c}\text { Total Hours Worked } \\
\text { including overtime }\end{array}$ & Total number of working hours including the extra work hours from direct operators \\
\hline $\begin{array}{c}\text { Number of Direct } \\
\text { Operators }\end{array}$ & $\begin{array}{c}\text { Total number of employees involved in the production process where the operations have } \\
\text { Standard Minute Values used for the calculation of Total Standard Hours produced }\end{array}$ \\
\hline $\begin{array}{c}\text { Efficiency of Direct } \\
\text { Operators }\end{array}$ & Total Number of Standard Hours produced/Total Hours Worked including overtime X 100\% \\
\hline $\begin{array}{c}\text { Utilization of direct } \\
\text { operators }\end{array}$ & (Total Hours Worked including overtime - Off-Standard Hours) / Total Hours Worked including \\
overtime (\%)
\end{tabular}


Table 4. Comparison of the Key Performance Indicators for the two design solutions

\begin{tabular}{|c|c|c|c|}
\hline Indicators & $\begin{array}{c}\text { Ease controlled 3D-to-2D } \\
\text { method }\end{array}$ & Method in this research & $\begin{array}{c}\text { 2D-to-3D virtual design } \\
\text { method }\end{array}$ \\
\hline $\begin{array}{c}\text { Total Hours Worked including } \\
\text { overtime }\end{array}$ & $10 \mathrm{~h}$ & $7.5 \mathrm{~h}$ & $12 \mathrm{~h}$ \\
\hline Number of Direct Operators & 1 (one designer) & 1 (one designer) & $\begin{array}{c}2 \text { (one designer and one } \\
\text { pattern maker) }\end{array}$ \\
\hline Efficiency of Direct Operators & $90 \%$ & $93 \%$ & $88 \%$ \\
\hline Utilization of direct operators & $93 \%$ & $95 \%$ & $83 \%$ \\
\hline Number of Indirect Operators & 0 & 0 & 1 \\
\hline Absenteeism & $9 \%$ & $9 \%$ & $12 \%$ \\
\hline
\end{tabular}

between the 2D technical parameters and 3D virtual garment try-on effects cannot be completely controlled. In conclusion, in terms of the efficiency, operation complexity, working cycle and resources occupation in the garment design and production process, the proposed 3D-to-2D virtual design method has obvious advantages than virtual design methods.

\section{Conclusion}

This paper presents a new virtual reality based method for designing customized garments, aimed to the physically disabled people with scoliosis. The virtual prototyping method is realized by the virtual human model created using a 3D body scanner, permitting to simulate the consumer's morphological shape with atypical physical deformations. Next, customized $2 \mathrm{D}$ and $3 \mathrm{D}$ virtual garment prototyping tools will be used to create products through interactions using fashion design and garment pattern design knowledge. The knowledge-based 3D garment design method is realized using the design knowledge and design process, which is already applied to normal body shapes successfully. Both the characters of the disabled people with scoliosis and the relationship between human body and garment are applied as the main principles in the prototyping process.

The proposed virtual prototyping method provides possibilities for the designers to design customized garment effectively. Both the beauty of the human body and comfort is taken into consideration in the prototyping process. A design case of a shirt was presented to show that the garment block design from the proposed method could be further applied to design garment products with various design elements.

The proposed method is validated by the comparison experiments with current design solutions for designing personalized garment products for physically disabled people with scoliosis. The result indicates that: firstly, the proposed method can solve the problem of customized design for disabled people with scoliosis; secondly, the proposed method ensures fast design and the design result can be applied for mass production; the design process is visible for the customers, which permits to quick interaction between the customers and designers to make the design process more efficient.

\section{References}

[1] Subramanyam R, Schaffzin J, Cudilo EM, Rao MB and Varughese AM. Systematic review of risk factors for surgical site infection in pediatric scoliosis surgery. Spine J. 2015; 15: 1422-31.

[2] Hong $Y$, Zeng $X$, Bruniaux $P$ and Liu K. Interactive virtual try-on based three-dimensional garment block design for disabled people of scoliosis type. Textile Research Journal. 2016: 0040517516651105.

[3] Y. HONG PB, X. ZENG1, K. LIU, M.DONG. Virtual Reality Based Collaborative Design Method for Designing Customized Garment of Disabled People with Scoliosis. International Journal of Clothing Science and Technology. 2017; 29.

[4] Hong $Y$, Curteza $A$, Zeng $X$, Bruniaux $P$ and Chen $Y$. Sensory evaluation based fuzzy AHP approach for material selection in customized garment design and development process. Book of Abstracts. lasi: IOP Publishing, 2016, p. 1-8.

[5] Luo ZG and Yuen MMF. Reactive 2D/3D garment pattern design modification. Computer-Aided Design. 2005; 37: 623-30.

[6] Liu Y-J, Zhang D-L and Yuen MM-F. A survey on CAD methods in $3 D$ garment design. Computers in Industry. 2010; 61: 576-93.

[7] HONG Y, ZENG $X$ and BRUNIAUX P. SELECTION AND APPLICATION OF KEY PERFORMANCE INDICATORS FORDESIGNAND PRODUCTION PROCESS. Uncertainty Modelling in Knowledge Engineering and Decision Making: Proceedings of the 12th International FLINS Conference. Roubaix: World Scientific, 2016, p. 1008-14.

[8] Sayem ASM, Kennon R and Clarke N. 3D CAD systems for the clothing industry. International Journal of Fashion Design, Technology and Education. 2010; 3: 45-53.

[9] Sayem ASM, Kennon $R$ and Clarke N. Resizable trouser template for virtual design and pattern flattening. International Journal of Fashion Design, Technology and Education. 2012; 5: 55-65.

[10] Zhang D, Wang J and Yang Y. Design 3D garments for scanned human bodies. Journal of Mechanical Science and Technology. 2014; 28: 2479-87.

[11] Thomassey S and Bruniaux P. A template of ease allowance for garments based on a $3 D$ reverse methodology. International Journal of Industrial Ergonomics. 2013; 43: 406-16. 
[12] Wang J, Lu G, Li W, Chen L and Sakaguti Y. Interactive 3D garment design with constrained contour curves and style curves. Computer-Aided Design. 2009; 41: 614-25.

[13] Wang CCL, Wang $Y$ and Yuen MMF. Design automation for customized apparel products. Computer-Aided Design. 2005; 37: 675-91.

[14] Volino P, Cordier F and Magnenat-Thalmann N. From early virtual garment simulation to interactive fashion design. Computer-Aided Design. 2005; 37: 593-608.

[15] Goldstein Y, Robinet P, Kartsounis G-A, et al. Virtual prototyping: from concept to $3 D$ design and prototyping in hours. Transforming Clothing Production into a DemandDriven, Knowledge-Based, High-Tech Industry. Springer, 2009, p. 95-139.

[16] WOOD F, Archibald L and Ewing W. THE LINRA SUNRAY CREASE-RECOVERY TEST. Journal of the Textile Institute Proceedings. 1962; 53: P135-P42.

[17] Stjepanovic Z. Computer-aided processes in garment production: features of CAD/CAM hardware. International Journal of Clothing Science and Technology. 1995; 7: 818.

[18] Shin K. Patternmaking for the underwired bra: New directions. Journal of the Textile Institute. 2007; 98: 30118.

[19] Huang H, Mok P, Kwok Yand Au J. Block pattern generation: From parameterizing human bodies to fit feature-aligned and flattenable $3 D$ garments. Computers in Industry. 2012; 63: 680-91.
[20] Hinds B, McCartney J and Woods G. Pattern development for 3D surfaces. Computer-Aided Design. 1991; 23: 58392.

[21] Efrat $S$. The development of a method for generating patterns for garments that conform to the shape of the human body. De Montfort University, 1982.

[22] Meng Y, Mok PY and Jin X. Computer aided clothing pattern design with $3 D$ editing and pattern alteration. Computer-Aided Design. 2012; 44: 721-34.

[23] Hsiao S-W and Chen R-Q. A study of surface reconstruction for $3 D$ mannequins based on feature curves. ComputerAided Design. 2013; 45: 1426-41.

[24] Jevsnik S, Pilar T, Stjepanovic Z and Rudolf A. Virtual prototyping of garments and their fit to the body. DAAAM International Scientific Book. 2012: 601-19.

[25] Fontana M, Rizzi C and Cugini U. 3D virtual apparel design for industrial applications. Computer-Aided Design. 2005; 37: 609-22.

[26] HONG Y, ZENG $X$ and BRUNIAUX P. KNOWLEDGE ACQUISITION AND MODELING OF GARMENT PRODUCT DEVELOPMENT. Uncertainty Modelling in Knowledge Engineering and Decision Making: Proceedings of the 12th International FLINS Conference (FLINS 2016). Roubaix: World Scientific, 2016, p. 438-44.

[27] Moon KK-L, Yi CY and Ngai EWT. An instrument for measuring supply chain flexibility for the textile and clothing companies. European Journal of Operational Research. 2012; 222: 191-203. 